\title{
A meta-analysis of Xin kai bitter method in the treatment of functional dyspepsia
}

\author{
Tao Yuan, Pei Li, Bo Jia \\ School of Basic Medical Sciences, Chengdu University of Traditional Chinese Medicine, Chengdu 611137, China \\ Contributions: (I) Conception and design: T Yuan, B Jia; (II) Administrative support: T Yuan, P Li; (III) Provision of study materials or patients: P Li, \\ B Jia; (IV) Collection and assembly of data: All authors; (V) Data analysis and interpretation: T Yuan, B Jia; (VI) Manuscript writing: All authors; (VII) \\ Final approval of manuscript: All authors. \\ Correspondence to: Bo Jia. School of Basic Medical Sciences, Chengdu University of Traditional Chinese Medicine, No. 37, Shierqiao Road, Chengdu \\ 611137, China. Email: jiabojiaboj@163.com.
}

Background: Functional dyspepsia (FD) is a common, etiologically complex disease which persistently and recurrently attacks the digestive system. However, the efficacy of Western medicine in treating FD is unsatisfactory and its use is often accompanied with severe side effects. Here, this study conducted a metaanalysis on the clinical efficacy and safety of the treatment of FD with Xin kai bitter method combined with Western medicine, to produce a more objective and comprehensive systematic review to guide clinical application.

Methods: Systematic searches were conducted of the PubMed, Cochrane Library, EMBASE, Medline, Web of Science, Wanfang Databases, and Weipu (VIP) databases, as well as China National Knowledge Infrastructure (CNKI). Randomized controlled clinical trials (RCTs) of Xin kai bitter method in the treatment of FD were included in the study. The total effective rate and safety were evaluated with relative risk (RR) and the quantitative data were evaluated with standard mean difference (SMD) and 95\% confidence interval (CI). The quality of the included literature was evaluated using RevMan5.3 software, and the "meta" package of R3.5.1 software was used for all other statistical analysis.

Results: A total of 24 papers involving 1,044 patients in the treatment group and 989 patients in the control group were included. A total of 23 articles reported the total effective rate after 1 month of treatment $\left(\mathrm{I}^{2}=0 \%\right)$, and the total effective rate in the treatment group was 1.21 times higher than that in the control group (95\% CI: 1.17 vs. 1.26). A total of 4 articles reported the safety rate after 1 month of treatment $\left(I^{2}=27 \%\right)$; the safety rate in the treatment group was 0.43 times than that in the control group (95\% CI: 0.23 vs. 0.82 ). A total of 8 articles reported traditional Chinese medicine (TCM) symptom score or clinical symptoms before and after 1 month of treatment $\left(\mathrm{I}^{2}=91 \%\right)$, and the difference in TCM symptom score before and after treatment in the treatment group was significantly lower than that in the control group, with a SMD of -1.19 (95\% CI: -1.71 , -0.66). A total of 6 articles reported the motilin (MTL) level before and after 1 month of treatment, and the difference before and after treatment in MTL in the treatment group was not significantly different to that in the control group, with a SMD of 0.92 (95\% CI: -0.12, 1.97).

Conclusions: Compared to conventional treatment, Xin kai bitter method has a higher clinical effect and lower adverse reaction rate in patients with FD, and can improve TCM symptom score. However, highquality RCT research is still needed to further explore the safety of Xin kai bitter method for treating FD.

Keywords: Functional dyspepsia (FD); Xin kai bitter method; Banxia Xiexin Decoction; meta-analysis

Submitted Mar 02, 2020. Accepted for publication Apr 24, 2020.

doi: $10.21037 /$ apm-20-860

View this article at: http://dx.doi.org/10.21037/apm-20-860 


\section{Introduction}

Functional dyspepsia (FD) is a common, etiologically complex disease which persistently and recurrently attacks the digestive system. FD clinically manifests as upper abdominal pain, postprandial fullness, flatulence, belching, nausea, and other symptoms in the upper abdomen (1). In Eastern countries, the prevalence rate of FD is about 5.3\% to $12.8 \%$ (2). In China specifically, FD patients comprise about $30 \%$ of patients at gastrointestinal specialist outpatient services. Lifestyle changes can form a part of treating FD, as can Western medicine, including gastric motility drugs, acid suppressants and anti-anxiety and anti-depression drugs, which can offer symptomatic relief (3). However, the efficacy of Western medicine in treating FD is unsatisfactory and its use is often accompanied by severe side effects. In comparison, Chinese medicine takes into account the integrity of prescriptions and uses a dialectical approach to medicines, leading to a better treatment effect (4). Chinese medicine categorizes FD into the categories such as "painful abdominal mass" or "epigastric pain", which reflects the belief by which it is underpinned; that the onset of FD is closely related to the spleen, stomach, and liver, and is caused by mixed heat and cold, nausea and vomiting, blood stasis and qi stagnation, and liver stagnation and qi stagnation. Xin kai bitter method is mainly derived from "Treatise on Febrile Diseases" and belongs to the category of "harmonization" method in Chinese medicine. It is pointed out in "The Yellow Emperor's Internal Canon of Medicine "that for Xin kai bitter method, Xin medicine has a medicinal property of yang, which can disperse and work, while bitter medicinal medicine belongs to yin, which can reduce energy and release it. The use of Xin kai bitter method can "drive heat and dehumidify," and "open air and turbidity." In the Xin kai bitter method, the main representative of which is the Banxia Xiexin Decoction, Xin hot is treated with ginger; the bitter cold is treated by astragalus and coptis; ginseng is added for sweet heat; along with licorice and jujube to nourish the spleen and stomach. The combination of hard bitter, cold, and warmth can play a role in cleansing, reducing turbidity, and releasing heat. This method is mainly used to treat cold and heat mixed types of FD (5).

At present, there are many obstacles in the way of establishing the best therapy for FD, including the small scale of most traditional Chinese medicine research, the rigorous research design, the low quality of the literature, and the inadequate digging of various empirical information contained in clinical data. Li et al. carried out a systematic review of Xin kai bitter method for FD treatment in 2015, mainly focusing on the treatment of FD with Banxia Xiexin Decoction, in which the experimental group was only treated with Chinese medicine (6). Subsequently, a large number of studies on the treatment of FD with integrated Chinese and Western medicine were carried out in China; however there has been no systematic evaluation of Xin kai bitter method combined with conventional Western medicine for FD treatment. Here, based on randomized controlled clinical trials, this study conducted a meta-analysis on the clinical efficacy and safety of the treatment of FD with Xin kai bitter method combined with Western medicine, to produce a more objective and comprehensive systematic review to guide clinical application. We present the following article in accordance with the PRISMA reporting checklist (available at http://dx.doi.org/10.21037/apm-20-860).

\section{Methods}

\section{Literature search}

We searched the English-language databases PubMed, Cochrane Library, EMBASE, Medline, and Web of Science for randomized controlled trials on FD conducted up to January 31, 2020. The search was limited to English and search terms included: "traditional Chinese medicine", "TCM", "Banxia Xiexin Decoction", "Xiexin Soup of Pinellia" and "Xin kai bitter method". Diseases terms contained "functional dyspepsia" and "FD". Searches were also conducted of the Chinese-language databases China National Knowledge Infrastructure (CNKI), Wanfang Database, and Wipp Database (VIP), and search terms included: "Xin Kai bitter method", "Banxia Xiexin Decoction", "Huanglian Decoction", "Weikang Ning", "Xiaopi Tongjiang Decoction", "Wumei Pill", and "Chest Decoction".

\section{Inclusion and exclusion criteria}

Studies that met the following criteria were included: (I) the diagnosis of FD was based on the Rome II or III standards, or the "Consensus Opinions for the Diagnosis and Treatment of Functional Western Dyspepsia plus Xin kai bitter method 2017" (7,8); (II) the intervention control group was treated with conventional Western medicine; (III) the test group was treated with Xin kai bitter method with conventional Western medicine as a control; (IV) the study was designed as a randomized 
clinical controlled trial; (V) the treatment course lasted 4 weeks or 1 month; (VI) outcome indicators included any of the following: total effective rate of treatment, incidence of adverse reactions, TCM symptom score or clinical symptoms, and motilin (MTL). Studies that met any of the following criteria were excluded: (I) animal experiments, reviews, case reports, conference abstracts, etc.; (II) focused on older people or FD combined with other serious diseases; (III) non-randomized group clinical trials; (IV) other treatments such as acupuncture applied during the treatment. In cases of duplicate publications, the most complete or most recent published publications were included.

\section{Literature screening and data extraction}

Literature screening was independently conducted by two data extraction staff. First, the titles, abstracts and then full texts were read against the inclusion and exclusion criteria. Next, a unified data extraction form was used for data extraction and verification for studies that met the exclusion criteria. The basic extracted data from the literature included the author's name, publication date, source of the research subjects, disease course, treatment methods, and analysis indicators of the control and experimental groups. The sample sizes of the control group and test group, along with their respective effective rates and adverse reaction indicators, and the number of effective cases and adverse reactions were extracted. The mean and standard deviation of TCM symptom score or clinical symptoms, and MTL level were extracted from the test group and control group before and after 1 month of treatment. Then, the mean and standard deviation of the difference before and after treatment were calculated according to the RevMan 5.1 manual.

\section{Literature quality evaluation}

Two researchers conducted the literature treatment evaluation using the Cochrane risk bias assessment tool to assess the risk of bias in six areas (9): (I) selection bias, including whether the random allocation method was described in detail and the hidden random allocation method was described; (II) implementation bias: whether researchers and subjects were blinded and effective information was provided for judging blindness; (III) measurement bias: whether the researchers' evaluation of the results was blind; (IV) follow-up bias: whether reasons for loss of follow-up and withdrawal were reported, ensuring the integrity of the data; $(V)$ report bias: whether it was possible to judge the results of selective reports; (VI) other bias: whether it was possible to assess the source of other biases that exist. Each index was evaluated using low, unclear, or high bias. Where inconsistency arose during the processes of literature screening, data extraction, and article quality evaluation, a third person would review them again.

\section{Statistical analysis}

The quality of the included literature was evaluated by RevMan5.3 software. The total effective and safety rates were evaluated with relative risk (RR) and the quantitative data was evaluated with standard mean difference (SMD) and $95 \%$ confidence interval (CI). Heterogeneity was evaluated by $\mathrm{I}^{2}$. When $\mathrm{I}^{2} \leq 50 \%$, a fixed effects model was used, while a random effects model was conducted for effect value estimation if $\mathrm{I}^{2}>50 \%$. Where heterogeneity existed between studies, sensitivity meta-analysis was performed after the elimination of the literature on a one-by-one basis. A funnel plot, Begg's rank correlation, and Egger's regression were used to carry out qualitative evaluation of publication bias. The effect value combination, forest map, publication bias, sensitivity analysis, and other analysis were analyzed using the "meta" package of R3.5.1 software.

\section{Results}

\section{Basic information of literature inclusion}

A total of 721 Chinese and English articles were retrieved. After the titles, abstracts, and full texts of the articles were read, 24 articles met the inclusion criteria (10-33). A flowchart of the literature search process was shown in Figure 1. A total of 1,044 patients with FD and 989 controls were included in this meta-analysis. Among the 24 articles, 20 articles were published after 2010, with only 4 articles were published before 2010 . The research populations were widely reported except in several autonomous regions. The main application of Xin kai bitter method was Banxia Xiexin Decoction, which was reported in 23 articles. The characteristics of the included literature are shown in Table 1.

\section{Literature quality evaluation}

All of the included articles used random allocation to avoid patient selection bias, but implementation bias was unclear. 


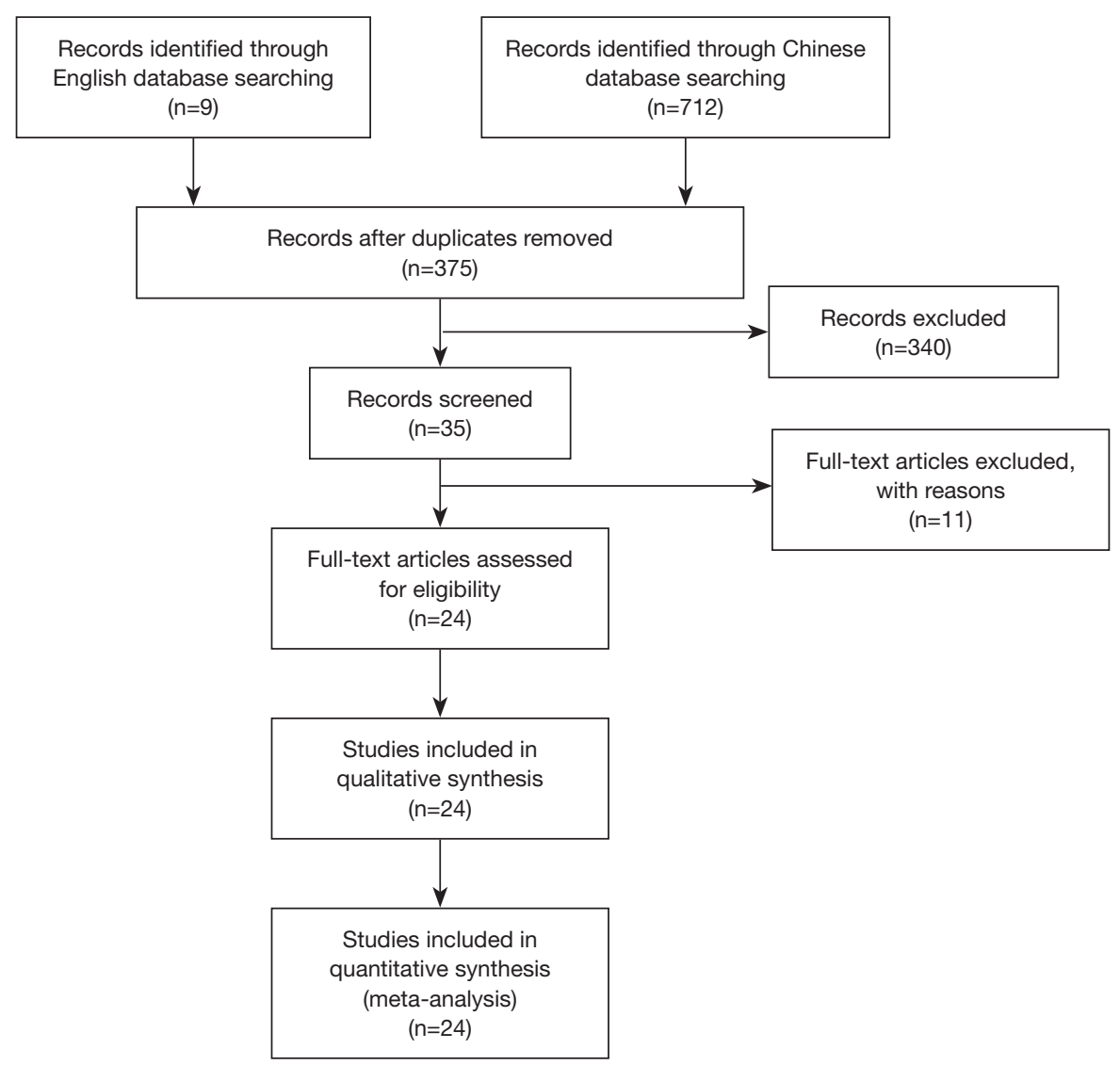

Figure 1 Literature search flowchart.

Table 1 Basic characteristics of the included literature

\begin{tabular}{|c|c|c|c|c|c|c|c|}
\hline Author & Year & Region & Course & $\begin{array}{l}\text { Control group } \\
\text { therapy }\end{array}$ & Test group therapy & $\begin{array}{l}\text { Control } \\
\text { population }\end{array}$ & $\begin{array}{l}\text { Test } \\
\text { population }\end{array}$ \\
\hline Yu & 2019 & Guangdong & 9 months -9 years & $\begin{array}{l}\text { Domperidone tablets } \\
\text { + compound azazolide } \\
\text { enteric-coated tablets } \\
\text { + mosapride citrate } \\
\text { dispersible tablets }\end{array}$ & $\begin{array}{l}\text { Plus Banxia Xiexin } \\
\text { Decoction }\end{array}$ & 52 & 52 \\
\hline Zhang & 2019 & Zhejiang & 6 months- 8 years & Domperidone & $\begin{array}{l}\text { Plus Banxia Xiexin } \\
\text { Decoction }\end{array}$ & 43 & 43 \\
\hline Yang & 2019 & Hunan & 4 months- 8 years & $\begin{array}{l}\text { Compound azintamide } \\
\text { enteric-coated tablets }\end{array}$ & $\begin{array}{l}\text { Plus Banxia Xiexin } \\
\text { Decoction }\end{array}$ & 48 & 48 \\
\hline Yang & 2019 & Shanxi & 6 months -5 years & Otilonium bromide tablets & $\begin{array}{l}\text { Plus Banxia Xiexin } \\
\text { Decoction }\end{array}$ & 25 & 25 \\
\hline
\end{tabular}

Table 1 (continued) 
Table 1 (continued)

\begin{tabular}{|c|c|c|c|c|c|c|c|}
\hline Author & Year & Region & Course & $\begin{array}{l}\text { Control group } \\
\text { therapy }\end{array}$ & Test group therapy & $\begin{array}{l}\text { Control } \\
\text { population }\end{array}$ & $\begin{array}{l}\text { Test } \\
\text { population }\end{array}$ \\
\hline Jin & 2019 & Jilin & $1-6$ weeks & $\begin{array}{l}\text { Mosapride citrate } \\
\text { dispersant tablet }\end{array}$ & $\begin{array}{l}\text { Plus Banxia Xiexin } \\
\text { decoction }\end{array}$ & 55 & 55 \\
\hline Zhao & 2018 & Sichuan & $1-8$ years & Domperidone & $\begin{array}{l}\text { Plus Banxia Xiexin } \\
\text { Decoction }\end{array}$ & 43 & 43 \\
\hline $\mathrm{Ma}$ & 2018 & Shaanxi & $0.5-7$ years & Mosapride tablets & $\begin{array}{l}\text { Plus Banxia Xiexin } \\
\text { Decoction }\end{array}$ & 49 & 49 \\
\hline Ding & 2018 & Henan & None & Deanxit + domperidone & $\begin{array}{l}\text { Plus Banxia Xiexin } \\
\text { Decoction }\end{array}$ & 45 & 45 \\
\hline Zhang & 2017 & Sichuan & $0.5-8$ weeks & Mosapride & $\begin{array}{l}\text { Plus Banxia Xiexin } \\
\text { Decoction }\end{array}$ & 40 & 40 \\
\hline $\mathrm{He}$ & 2016 & Guizhou & None & Domperidone & $\begin{array}{l}\text { Plus Banxia Xiexin } \\
\text { Decoction }\end{array}$ & 48 & 48 \\
\hline Tang & 2014 & Jiangxi & 3 months -3.9 years & $\begin{array}{l}\text { Domperidone + omeprazole } \\
\text { enteric-coated capsules }\end{array}$ & $\begin{array}{l}\text { ePlus Banxia Xiexin } \\
\text { Decoction }\end{array}$ & 29 & 29 \\
\hline Liu & 2013 & Hunan & None & Cisapilli + delixin & $\begin{array}{l}\text { Plus Xiaoyao powder + } \\
\text { Banxia Xiexin Decoction }\end{array}$ & 38 & 34 \\
\hline $\mathrm{He}$ & 2012 & Hubei & $1-8$ years & Mosapride & $\begin{array}{l}\text { Plus Banxia Xiexin } \\
\text { decoction }\end{array}$ & 62 & 62 \\
\hline Zhao & 2007 & Tianjin & $1.5-4$ years & Prepulsid & $\begin{array}{l}\text { Plus flavored small chest } \\
\text { soup }\end{array}$ & 32 & 30 \\
\hline Yao & 2007 & Guangdong & 6 weeks-8 years & Mosapride & $\begin{array}{l}\text { Plus Banxia Xiexin } \\
\text { Decoction }\end{array}$ & 53 & 29 \\
\hline Yan & 2004 & Beijing & Unknown & $\begin{array}{l}\text { Modisulin tablets + xia shen } \\
\text { and stomach capsules } \\
\text { simulation capsules }\end{array}$ & $\begin{array}{l}\text { nGinseng and stomach } \\
\text { capsules + modyline } \\
\text { analog tablets }\end{array}$ & 20 & 20 \\
\hline Huang & 2004 & Guangxi & 1 months -15 years & Morpholine & $\begin{array}{l}\text { Plus Banxia Xiexin } \\
\text { Decoction }\end{array}$ & 60 & 63 \\
\hline
\end{tabular}




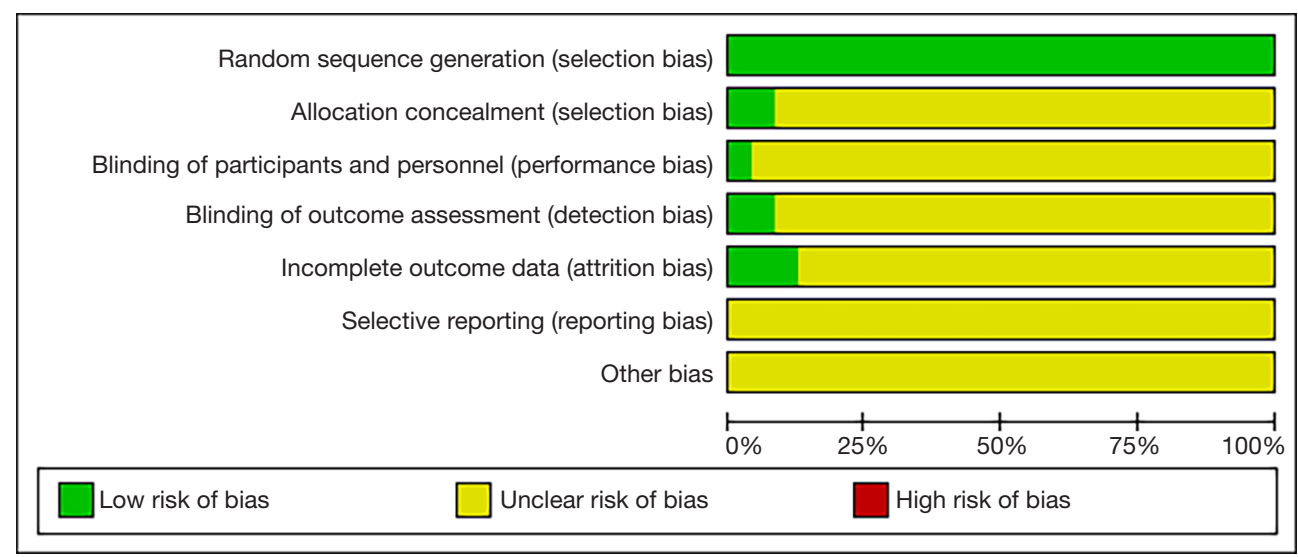

Figure 2 Evaluation of quality bias of the included literature.

Only one literature used a double-blind method (32). Three articles described the random allocation method in detail and reported the completeness of follow-up results $(11,22,32)$. Follow-up bias, reporting bias, and other aspects of bias in the included articles were not clear. The Cochrane risk-of-bias assessment is shown in Figure 2.

\section{Meta-analysis of effective rate}

Of the articles, 23 reported the total effective rate after 1 month of treatment $\left(\mathrm{I}^{2}=0 \%\right)$. The RR was estimated using a fixed effects model, and the total effective rate of the experimental group was found to be 1.21 times (95\% CI: $1.17,1.26$ ) that of the control group (Figure 3).

\section{Meta-analysis of adverse reactions}

Four articles reported the safety rate after 1 month of treatment $\left(I^{2}=27 \%\right)$. The $R R$ was estimated using a fixed effects model to calculate the safety rate, which was 0.43 times higher in the treatment group than in the control group $(95 \%$ CI: 0.23 vs. 0.82) (Figure 4).

\section{Meta-analysis of TCM symptom score}

Eight articles reported TCM symptom score or clinical symptoms before and after 1 month of treatment $\left(I^{2}=91 \%\right)$. A random effects model was used to estimate SMD. The difference in TCM symptom score before and after treatment in the treatment group was significantly lower than that in the control group (SMD: $-1.19 ; 95 \%$ CI: -1.71 , -0.66 ; Figure 5).

\section{Meta-analysis of MLT}

Six articles reported MLT before and after 1 month of treatment $\left(\mathrm{I}^{2}=97 \%\right)$. A random effects model was used to estimate SMD. No significant difference in MLT was found between the treatment and control groups before and after treatment (SMD: 0.92; 95\% CI: -0.12, 1.97; Figure 6).

\section{Sensitivity analysis and publication bias evaluation}

Sensitivity analysis was performed after removing the articles one by one. Total effectiveness, safety rate, and TCM symptom score were stable with no significant change. However, when the report by Xie et al. was excluded (27), the sensitivity analysis indicated that the difference of MLT in the experimental was significantly higher than in the control group (SMD: 1.42; 95\% CI: 0.78, 2.07; Figure 7). Egger's regression and Begg's rank correlation analysis indicated that apart from statistical differences in efficiency and TCM symptom score, there was no publication bias found in other studies ( $\mathrm{P}>0.05$; Table 2$)$.

\section{Discussion}

FD, a common heterogeneous disease with complex etiology, adversely impacts patients' health, work, and quality of life due to its chronic and recurrent attacks. In addition to conventional support therapies such as healthy lifestyle changes, maintaining a calm mind, and keeping a regular diet, FD patients often receive clinical therapy in the form of drugs, such as domperidone, cisapride, and mosapride, which promote gastric motility. Another 




Figure 3 Meta-analysis forest map of the total effective rate of Xin kai bitter method for the treatment of FD. FD, functional dyspepsia.

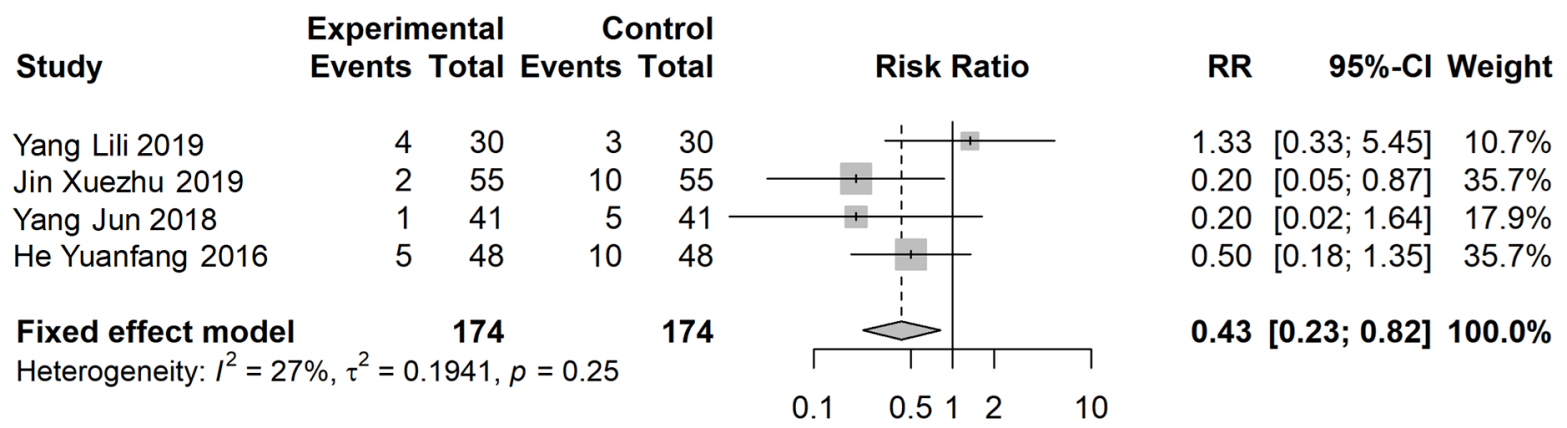

Figure 4 Meta-analysis forest map of the incidence of adverse reactions of FD treated with Xin Kai bitter method. FD, functional dyspepsia.

clinical practice is antacid treatment, like omeprazole, and antidepressant drugs, such as dilixin. Currently, the efficacy of Western medicine alone for treating FD is not clear, while its higher price, especially over the long term, can result in poor compliance among patients.

In Chinese medicine, with its dialectical nature, FD is divided into five types of syndromes: liver-qi stagnation, liver-qi attacking the stomach, spleen deficiency and stomach qi, damp-heat stagnation stomach syndrome, and cold and heat mixed complex lesions. The treatments for these different syndromes are slightly different. In traditional Chinese medicine, Xin kai bitter method mainly comes from "Treatise on Febrile Diseases" and belongs to the category of "Regulating therapy" (34). In "Treatment of Febrile and Miscellaneous Diseases", there are prescriptions such as Xiexin Decoction and Decoction of Chest in which 


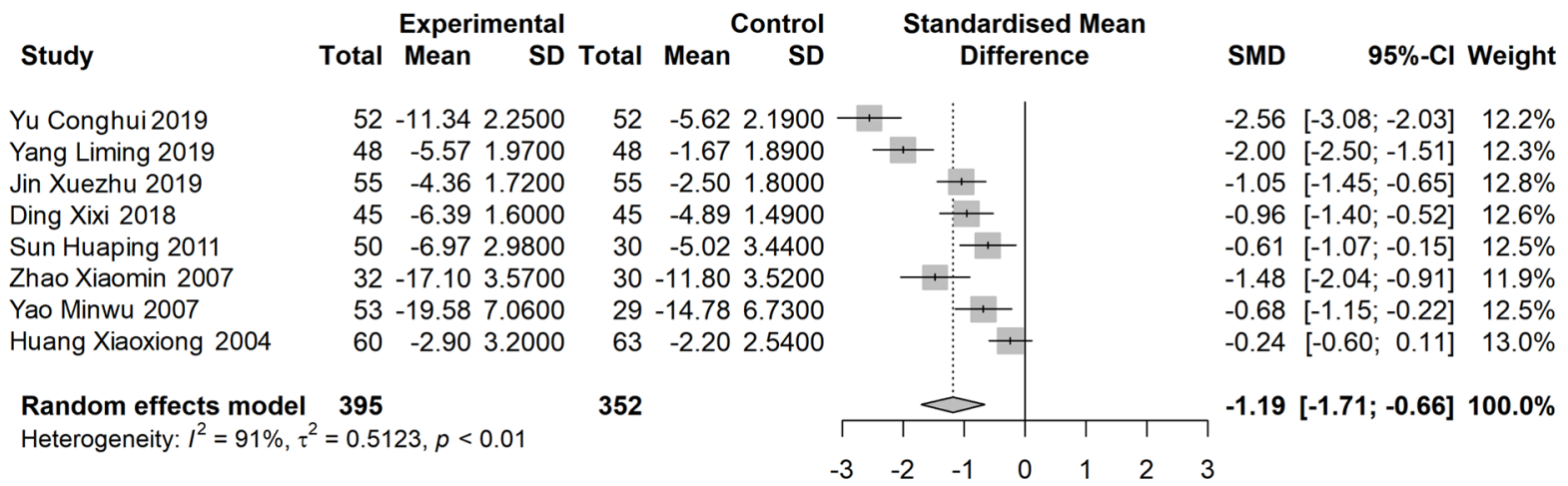

Figure 5 Meta-analysis forest map of the difference in TCM symptom score before and after FD treatment. FD, functional dyspepsia.



Figure 6 Meta-analysis forest map of the difference in MLT before and after FD treatment. MLT, motilin; FD, functional dyspepsia.



Figure 7 Sensitivity analysis of the difference in MLT before and after FD treatment. MLT, motilin; FD, functional dyspepsia.

Xin kai bitter method is used for cold and heat mixed complex lesions in the spleen and stomach, poor ventilator disease, diabetes, and lung and heart diseases. Banxia Xiexin Decoction comes from "Treatment of Febrile and Miscellaneous Diseases" and is one of the representatives of the Xin kai bitter method for treating cold and heat mixed syndrome of FD. In this prescription, pinellia and dried ginger are used to warm the stomach and disperse cold. Coptis chinensis and radix scutellariae are bitter and detoxify and release heat from the body and relieve the qi in stomach. Pinellia is compatible with coptis chinensis for reducing nausea and enhancing the harmony of the stomach. The combination of cinnamon sticks, dried ginger, radix scutellariae, and coptis chinensis can clear heat and cold. 
Table 2 Meta-analysis of Xin Kai bitter method for FD

\begin{tabular}{lccccc}
\hline \multirow{2}{*}{ Category } & \multicolumn{3}{c}{ Egger's } & & Begg's \\
\cline { 2 - 5 } & $\mathrm{t}$ & $\mathrm{P}$ & $\mathrm{Z}$ & $\mathrm{P}$ \\
\hline Effective rate & 5.332 & $<0.001$ & 4.094 & $<.001$ \\
Adverse reactions rate & -0.535 & 0.646 & -0.679 & 0.497 \\
TCM syndrome score & -2.673 & 0.037 & -1.732 & 0.083 \\
MLT & -0.073 & 0.945 & 0.564 & 0.573 \\
\hline
\end{tabular}

MLT, motilin; FD, functional dyspepsia.

Ginseng has a strengthening effect on the spleen and nourishes qi, while angelica can nourish blood and qi. The combination of jujube, licorice, and ginseng can nourish the spleen and stomach and combined with the medicines above, can restore the spleen and stomach and neutralize the deficiency. The combination of various medicines can be used to supplement each other to strengthen the spleen and stomach, regulate the Qi activity, warm the spleen and clear cold, and nourish the liver and release depression (34).

In this study, the total effective rate of conventional Western medicine combined with Xin kai bitter method for treating FD was 1.21 times (95\% CI: 1.17, 1.26) higher than that in the control group, and the incidence of adverse reactions was 0.43 times $(95 \% \mathrm{CI})$ that in the control group (95\% CI: 0.23, 0.82), which indicated Xin kai bitter method to have a significantly better effect in FD than conventional Western medicine. The application of Banxia Xiexin Decoction is based on the different symptoms of FD patients and it is added to or subtracted from prescriptions accordingly. It has a superior effect on individualized treatment and the alleviation of patients' symptoms and improves the treatment effect. Recent studies have confirmed that Pinellia ternata can inhibit the secretion of gastric acid and astragalus can enhance the body's resistance to disease. In addition, flavonoids and terpenoids in licorice can regulate the immune system and have an anti-inflammatory effect by reducing the level of cellular inflammatory factors (18). Therefore, the combination of Xin kai bitter method and Western medicine such as domperidone has the function of treating visceral dysfunction and strengthening the spleen, but without the adverse reactions caused by Western medicine, such as diarrhea, exhaustion, and dizziness.

After treatment with conventional Western medicine plus Xin kai bitter method, the TCM symptom score decreased, and the difference in the experimental group was significantly lower than that in the control group (SMD: $-1.19 ; 95 \%$
CI: $-1.71,-0.66)$. MLT increased after treatment with conventional Western medicine plus Xin kai bitter method or conventional Western medicine only, and there was no significant difference in the difference of MLT elevation before and after treatment in the two groups (SMD: 0.92; $95 \%$ CI: $-0.12,1.97)$. Remarkably, the sensitivity analysis found that after the deletion of Xie Xiaoling's study (27), the MLT difference before and after treatment with conventional Western medicine plus Xin kai bitter method was higher than the that in the control group (SMD: 1.42; 95\% CI: $0.78,2.07)$. Through further analysis of the literature, we found that the research quality of this article was relatively poor owing to inconsistency between the table data and the descriptions given in the article. For the purpose of accuracy, we chose to use the data in the tables. Therefore, the conclusion that MLT levels are increased with treatment with conventional Western medicine plus Xin Kai Kuo method must be cautiously drawn.

Through Egger's regression and Begg's rank correlation, it was indicated that except for statistical differences in the efficiency and TCM symptom score, there was no publication bias found in the other studies. However, there were only 4 articles with TCM syndrome score. When only a small number of studies are being considered, the test efficiency is too low to sufficiently identify publication bias using funnel charts. Studies have shown that when the number of studies in the literature was less than 10 , the results of the publication bias assessment were unstable, and it was recommended not to conduct a publication bias analysis. However, an examination of treatment efficiency suggested that a publication bias existed, meaning that some studies were possibly unpublished that indicates conventional Western medicine plus Xin kai bitter method is superior to Western medicine.

The analysis of TCM syndrome score and MLT was relatively heterogeneous, which might be attributed to the following reasons: (I) differences in reagents and equipment 
used by hospitals for MLT measurement; (II) variations in patient characteristics across different regions, for example, the course lasted for only a few weeks in some studies (20), while in some other studies the shortest course of was 1.5 years (30); (III) a lack of uniformity in the treatment method of Western medicine used in the control group; and (IV) slight differences in the composition and measurement of the prescriptions used in the Western medicine plus Xin kai bitter method group. However, due to the limited literature data, the data were not comprehensive enough to support further hierarchical analysis.

This meta-analysis had the following limitations: (I) most of the included articles were of poor quality, and only one article (32) described the randomized double-blind method. There were no reports on selection bias, implementation bias, or measurement bias, so the research bias cannot be ruled out; (II) most of the literature in this study did not explain the TCM syndromes of FD patients. Because dialectical treatment is the principle of TCM, and Banxia Xiexin Decoction mainly treats cold and heat mixed complex lesions, large samples, randomized double-blind clinical trials and strict dialectical treatment of TCM syndromes in FD patients were needed to further confirm our results.

In summary, this meta-analysis found that, based on the research samples, conventional Western medicine plus Xin kai bitter method is effective in treating FD, with a low rate of adverse reactions, and can effectively improve the clinical symptoms of patients and reduce TCM symptom score. However, due to the limitations of this meta-analysis, including low quality of the included articles and their small sample sizes, randomized double-blind clinical trials with large sample sizes and studies strictly on TCM syndromes in FD patients need to be carried out to confirm the results, especially with regard to the safety of the long-term cited drugs, to comprehensively assess the effectiveness and safety of Xin kai bitter method for treating patients with FD.

\section{Acknowledgments}

Funding: None.

\section{Footnote}

Reporting Checklist: The authors have completed the PRISMA reporting checklist. Available at http://dx.doi. org/10.21037/apm-20-860

Conflicts of Interest: All authors have completed the ICMJE uniform disclosure form (available at http://dx.doi. org/10.21037/apm-20-860). The authors have no conflicts of interest to declare.

Ethical Statement: The authors are accountable for all aspects of the work in ensuring that questions related to the accuracy or integrity of any part of the work are appropriately investigated and resolved.

Open Access Statement: This is an Open Access article distributed in accordance with the Creative Commons Attribution-NonCommercial-NoDerivs 4.0 International License (CC BY-NC-ND 4.0), which permits the noncommercial replication and distribution of the article with the strict proviso that no changes or edits are made and the original work is properly cited (including links to both the formal publication through the relevant DOI and the license). See: https://creativecommons.org/licenses/by-nc-nd/4.0/.

\section{References}

1. Satsangi AK, Brugnoli MP. Anxiety and psychosomatic symptoms in palliative care: from neuro-psychobiological response to stress, to symptoms' management with clinical hypnosis and meditative states. Ann Palliat Med 2018;7:75-111.

2. Prado BL, Qian Y. Anti-cytokines in the treatment of cancer cachexia. Ann Palliat Med 2019;8:67-79.

3. Madisch A, Andresen V, Enck P, et al. The Diagnosis and Treatment of Functional Dyspepsia. Dtsch Arztebl Int 2018;115:222-32.

4. Lai Y, Liu Y, He Y, et al. Clinical observation on Xiaopi Tongjiang decoction in treating mixed functional dyspepsia. Hebei J of TCM 2019;41:220-3.

5. Luan Y, Zhang W, Yan J. Research Progress of the Method of Using Acridity to Open and Bitterness to Discharge. Journal of New Chinese Medicine 2019;51:31-6.

6. Li S, Li D. Systematic review of Ban-xia xie-xin Decoction for the treatment of functional dyspepsia. Beijing Journal of Traditional Chinese Medicine 2019;51:31-6.

7. Digestive Diseases Committee of China Society of Integrated Traditional Chinese and Western Medicine. Consensus on diagnosis and treatment of functional dyspepsia with integrated traditional Chinese and western medicine (2017). Chin J Integr Trad and West Med Dig 2017;25(12).

8. $\mathrm{Mu} \mathrm{H}$. Comparison of Rome III Criteria and Rome II Criteria in the Diagnosis of Functional Dyspepsia. Medical Information 2011;24(10). 
9. Higgins JPT, Green S. Cochrane Handbook for Systematic Reviews of Interventions Version 5.1.0 (EB/OL). The Cochrane Collaboration 2011 (2012-03-30). Available online: http://handbook-5-1.cochrane.org

10. Zhang S. Effect of Ban-xia xie-xin Decoction on Functional Dyspepsia. Special Health 2019;(19):215.

11. Zhang H, Chen H, Luo J. Clinical Study on Modified Banxia Xiexin Tang Combined with Domperidone for Functional Dyspepsia. Journal of New Chinese Medicine 2019;51:59-61.

12. Yu C, Hui Y, Jie Y. Observation on curative effect of combined Chinese and western medicine on functional dyspepsia. Journal of Practical Traditional Chinese Medicine 2019;35:193-4.

13. Yang L. Clinical Observation of Ban-xia xie-xin Decoction in Treating Functional Dyspepsia. Yunnan Journal of Traditional Chinese Medicine and Materia Medica 2019;40:100-1.

14. Yang L, Yao Y. Ban-xia xie-xin Decoction Combined with Mosapride in the Treatment of Functional Dyspepsia for 30 Cases. CJGMCM 2019;34:1102-4.

15. Yang Fan. Observation on treating 50 cases of functional dyspepsia with the Ban-xia xie-xin Decoction. CJCM 2019;11:112-3.

16. Jin X. Analysis of clinical value of Ban-xia xie-xin Decoction in the treatment of functional dyspepsia. Health and Guide 2019;263.

17. Zhao M. Clinical effect analysis of Ban-xia xie-xin Decoction in the treatment of functional dyspepsia with cold and heat syndromes. Health Way 2018.

18. Yang Jun. Effect of Ban-xia xie-xin Decoction on Functional Dyspepsia. Contemporary Medical Symposium 2018;16:209-10.

19. Ma X, Shi L, Zhang J. Clinical curative effect of Banxia xie-xin Decoction combined with Mosapride in the treatment of functional dyspepsia and its effect on plasma motilin. Shaanxi Journal of Traditional Chinese Medicine 2018;39:1399-401.

20. Lv X. Effect of Ban-xia xie-xin Decoction on Functional Indigestion. Modern Diagnosis \& Treatment 2018;29:2211-3.

21. Ding X. Clinical efficacy and safety observation of Ban-xia xie-xin Decoction in the treatment of functional dyspepsia. World Latest Medicine Information 2018;18:127.

22. Zhang Y. Ban-xia xie-xin Decoction combined with Mosapride in the Treatment of Functional Dyspepsia Randomized Controlled Study. Journal of Practical Traditional Chinese Internal Medicine 2017;31:73-5.
23. He Y, Tian K, Zheng Q. Clinical effect of traditional Chinese medicine on functional dyspepsia. The World Clinical Medicine 2016;10:150.

24. Tang L. Observation on the efficacy of Ban-xia xie-xin Decoction in treating functional dyspepsia. Journal of Mudanjiang Medical University 2014;35:80-1.

25. Liu Wei, Zhou Xiao. Clinical observation of combined traditional Chinese and western medicine in treating functional dyspepsia. CJGMCM 2013;28:1001-2.

26. He R, Xie X. Observation of Curative Effect of Mosapride and Ban-xia xie-xin Decoction on Functional Indigestion and Its Effect on Plasma Motilin. Journal of New Chinese Medicine 2012;44:39-40.

27. Xie X, Zhang L, Peng Y. Ban-xia xie-xin Decoction in Adjuvant Treatment of Functional Dyspepsia: A Report of 42 Cases. Journal of Anhui TCM College 2011;30:30-2.

28. Sun H. Clinical analysis of 50 cases of functional dyspepsia. Guide of China Medicine 2011;9:90-1.

29. Huang J. Observation on the efficacy of Ban-xia xiexin Decoction combined with moxapride citrate in the treatment of functional dyspepsia. Journal of Practical Traditional Chinese Medicine 2011;27:464-65.

30. Zhao X, Du X. Therapeutic effect of Jiawei Xiaozhan Decoction on 32 patients with functional dyspepsia. Lishizhen Medicine and Materia Medical Research 2007;1741.

31. Yao M. Effect of the Chinese Medicine Combined with Western Medicine Therapy on Functional Dyspepsia. Research and Practice of Chinese Medicines 2007;56-8.

32. Yan T. Clinical and Theoretical Study of Xia Shen He Wei Capsule for Functional Dyspepsia. Chinese Academy of Chinese Medical Sciences; China Academy of traditional Chinese Medicine 2004.

33. Huang X, Long J. Clinical Observation of Ban-xia xiexin Decoction Combined with Western Medicine for Functional Dyspepsia. Acta Medicinae Sinica 2004;17: 559-60.

34. Wang B, Su X, Zhang X. Evaluation on the efficacy and safety of pungent-opening and bitter-descending method in the treatment of functional dyspepsia. China Medical Herald 2016;13:112-7.

Cite this article as: Yuan T, Li P, Jia B. A meta-analysis of Xin kai bitter method in the treatment of functional dyspepsia. Ann Palliat Med 2020;9(3):993-1003. doi: 10.21037/apm-20-860 